\title{
Time transformation and reversibility of Nambu-Poisson systems
}

\author{
Klas MODIN ${ }^{a, b}$ \\ ${ }^{a}$ Centre for Mathematical Sciences, Lund University, Box 118, SE-221 00 Lund, Sweden \\ E-mail:kmodin@maths.lth.se \\ ${ }^{b}$ SKF Engineering \& Research Centre, MDC, RKs-2, SE-415 50 Göteborg, Sweden
}

\begin{abstract}
A time transformation technique for Nambu-Poisson systems is developed, and its structural properties are examined. The approach is based on extension of the phase space $\mathscr{P}$ into $\mathscr{P}=\mathscr{P} \times \mathbb{R}$, where the additional variable controls the time-stretching rate. It is shown that time transformation of a system on $\mathscr{P}$ can be realised as an extended system on $\overline{\mathscr{P}}$, with an extended Nambu-Poisson structure. In addition, reversible systems are studied in conjunction with the Nambu-Poisson structure. The application in mind is adaptive numerical integration by splitting of Nambu-Poisson Hamiltonians. As an example, a novel integration method for the rigid body problem is presented and analysed.
\end{abstract}

2000 MSC: 37M99, 22E70, 53Z05, 34A26

\section{Introduction}

In 1973 Nambu [23] suggested a generalisation of Hamiltonian mechanics, taking the Liouville condition on volume preservation in phase space as a governing principle. Nambu postulated that the governing equations for a dynamical system on $\mathbb{R}^{n}$ should have the form

$$
\frac{\mathrm{d} x_{i}}{\mathrm{~d} t}=\sum_{j_{1}, \ldots, j_{n-1}} \epsilon_{i j_{1} \ldots j_{n-1}} \frac{\partial H_{1}}{\partial x_{j_{1}}} \frac{\partial H_{2}}{\partial x_{j_{2}}} \ldots \frac{\partial H_{n-1}}{\partial x_{j_{n-1}}}
$$

where $\epsilon$ is the Levi-Civita tensor over $n$ indices, and $H_{1}, \ldots, H_{n-1}$ are smooth real valued functions on $\mathbb{R}^{n}$ called Hamiltonian functions. Notice that the vector field in equation (1.1) is source free (its divergence is zero), which implies that the corresponding phase flow is volume preserving.

Later Takhtajan [27] formalised Nambu's framework by introducing the concept of NambuPoisson brackets on general phase space manifolds. Based on Takhtajan's work the geometry of Nambu-Poisson structures has been explored in several papers [6, 4, 5, 21, 11, 22, 28, 29].

In this paper we study time transformation of Nambu-Poisson systems. Such transformations are important in the construction and analysis of adaptive structure preserving numerical time stepping methods $[26,10,3,7,24,18,2,20,19]$. The idea is to obtain time step adaptivity by equidistant discretisation in the transformed variable, which corresponds to non-equidistant discretisation in the original time variable. Although numerical integration is a main motivation, the focus in the paper is not on numerical issues, but rather on structural properties.

The current section continues with a brief review of Nambu-Poisson mechanics, and of a time transformation method by Hairer and Söderlind [9]. The main results are in Section 2, where time transformation for Nambu-Poisson systems is developed. In Section 3, the Nambu-Poisson 
Klas Modin

structure is studied in conjunction with reversibility. As an application, we show in Section 4 how to construct fully explicit, adaptive numerical integration methods based on splitting of the Nambu-Poisson Hamiltonians. In particular, a novel method for the free rigid body. Conclusions are given in Section 5 .

We adopt the following notation. $\mathscr{P}$ denotes a phase space manifold of dimension $n$, with local coordinates $\boldsymbol{x}=\left(x_{1}, \ldots, x_{n}\right)$. The algebra of smooth real valued functions on $\mathscr{P}$ is denoted $\mathcal{F}(\mathscr{P})$. Further, $\mathfrak{X}(\mathscr{P})$ denotes the linear space of vector fields on $\mathscr{P}$. The Lie derivative along $X \in \mathfrak{X}(\mathscr{P})$ is denoted $\mathcal{L}_{X}$. If $X, Y \in \mathfrak{X}(\mathscr{P})$ then the commutator $[X, Y]=\mathcal{L}_{X} Y$ supplies $\mathfrak{X}(\mathscr{P})$ with an infinite dimensional Lie algebra structure. Its corresponding Lie group is the set $\operatorname{Diff}(\mathscr{P})$ of diffeomorphisms on $\mathscr{P}$, with composition as group operation. (See McLachlan and Quispel [16] and Schmid [25] for issues concerning infinite dimensional Lie groups.) If $\Phi \in \operatorname{Diff}(\mathscr{P})$ then $\Phi^{*}$ denotes the pull-back map and $\Phi_{*}$ the push-forward map imposed by $\Phi$.

\subsection{Nambu-Poisson mechanics}

In Hamiltonian mechanics, the phase space manifold $\mathscr{P}$ is equipped with a Poisson structure, defined by a bracket operation $\left\{\cdot_{1}, \cdot_{2}\right\}: \mathcal{F}(\mathscr{P}) \times \mathcal{F}(\mathscr{P}) \rightarrow \mathcal{F}(\mathscr{P})$ that is skew-symmetric, fulfils the Leibniz rule and the Jacobi identity. Nambu-Poisson mechanics is a generalisation.

Definition 1.1. A Nambu-Poisson manifold of order $k$ consists of a smooth manifold $\mathscr{P}$ together with a multilinear map

$$
\left\{\cdot{ }_{1}, \ldots, \cdot{ }_{k}\right\}: \underbrace{\mathcal{F}(\mathscr{P}) \times \ldots \times \mathcal{F}(\mathscr{P})}_{k \text { times }} \rightarrow \mathcal{F}(\mathscr{P})
$$

that fulfils:

- total skew-symmetry

$$
\left\{H_{1}, \ldots, H_{k}\right\}=\operatorname{sgn}(\sigma)\left\{H_{\sigma_{1}}, \ldots, H_{\sigma_{k}}\right\}
$$

- Leibniz rule

$$
\left\{G H_{1}, \ldots, H_{k}\right\}=G\left\{H_{1}, \ldots, H_{m}\right\}+H_{1}\left\{G, H_{2}, \ldots, H_{k}\right\}
$$

- fundamental identity

$$
\begin{aligned}
\left\{H_{1}, \ldots, H_{k-1},\left\{G_{1}, \ldots, G_{k}\right\}\right\}= & \left\{\left\{H_{1}, \ldots, H_{k-1}, G_{1}\right\}, G_{2}, \ldots, G_{k}\right\} \\
& +\left\{G_{1},\left\{H_{1}, \ldots, H_{k-1}, G_{2}\right\}, G_{3}, \ldots, G_{k}\right\}+\ldots \\
& +\left\{G_{1}, \ldots, G_{k-1},\left\{H_{1}, \ldots, H_{k-1}, G_{k}\right\}\right\}
\end{aligned}
$$

Remark 1.1. The case $k=2$ coincides with ordinary Poisson manifolds.

The first two conditions, total skew-symmetry (1.2a) and Leibniz rule (1.2b), are straightforward: they imply that the bracket is of the form

$$
\left\{H_{1}, \ldots, H_{k}\right\}=\eta\left(\mathrm{d} H_{1}, \ldots, \mathrm{d} H_{k}\right)
$$


for some totally skew-symmetric contravariant $k$-tensor $\eta[27]$. The third condition, the fundamental identity (1.2c), is more intricate. The range of possible Poisson-Nambu brackets is heavily restricted by this condition [27].

A Nambu-Poisson system on a Nambu-Poisson manifold of order $k$ is determined by $k-1$ Hamiltonian function $H_{1}, \ldots, H_{k-1} \in \mathcal{F}(\mathscr{P})$. The governing equations are

$$
\frac{\mathrm{d} F}{\mathrm{~d} t}=\left\{H_{1}, \ldots, H_{k-1}, F\right\} \quad \forall F \in \mathcal{F}(\mathscr{P})
$$

which may also be written

$$
\frac{\mathrm{d} \boldsymbol{x}}{\mathrm{d} t}=X_{H_{1}, \ldots, H_{k-1}}(\boldsymbol{x})
$$

where $X_{H_{1}, \ldots, H_{k-1}} \in \mathfrak{X}(\mathscr{P})$ is defined by $\mathcal{L}_{X_{H_{1}, \ldots, H_{k-1}}} F=\left\{H_{1}, \ldots, H_{k-1}, F\right\}$. The corresponding flow map is denoted $\varphi_{H_{1}, \ldots, H_{k-1}}^{t}$. Notice that due to skew symmetry of the bracket, all the Hamiltonians $H_{1}, \ldots, H_{k-1}$ are first integrals, which follows from equation (1.3a).

Due to the fundamental identity (1.2c), Nambu-Poisson systems fulfil certain properties which have direct counterparts in Hamiltonian mechanics (the case $k=2$ ).

Theorem 1.1 (Takhtajan [27]). The set of first integrals of system (1.3) is closed under the Nambu-Poisson bracket. That is, if $G_{1}, \ldots, G_{k}$ are first integrals, then $\left\{G_{1}, \ldots, G_{k}\right\}$ is again a first integral.

Theorem 1.2 (Takhtajan [27]). The flow of system (1.3) preserves the Nambu-Poisson structure. That is,

$$
\left\{G_{1}, \ldots, G_{k}\right\} \circ \varphi_{H_{1}, \ldots, H_{k-1}}^{t}=\left\{G_{1} \circ \varphi_{H_{1}, \ldots, H_{k-1}}^{t}, \ldots, G_{n} \circ \varphi_{H_{1}, \ldots, H_{k-1}}^{t}\right\} \quad \forall G_{1}, \ldots, G_{k} \in \mathcal{F}(\mathscr{P})
$$

or equivalently

$$
\mathcal{L}_{X_{H_{1}, \ldots, H_{k-1}}} \eta=0
$$

Remark 1.2. The set of vector fields that fulfils equation (1.4) is denoted $\mathfrak{X}_{\eta}(\mathscr{P})$. Clearly $\mathfrak{X}_{\eta}(\mathscr{P})$ is closed under linear combinations, so it is a sub-space of $\mathfrak{X}(\mathscr{P})$. Further, since $\mathcal{L}_{[X, Y]} \eta=$ $\mathcal{L}_{X}\left(\mathcal{L}_{Y} \eta\right)-\mathcal{L}_{Y}\left(\mathcal{L}_{X} \eta\right)$ it is also closed under the commutator. Thus, $\mathfrak{X}_{\eta}(\mathscr{P})$ is a Lie sub-algebra of $\mathfrak{X}(\mathscr{P})$. Correspondingly, $\operatorname{Diff}_{\eta}(\mathscr{P})$ denotes the Lie sub-group of $\operatorname{Diff}(\mathscr{P})$ that preserves the Nambu-Poisson structure. An element $\Phi \in \operatorname{Diff}_{\eta}(\mathscr{P})$ is called an $\eta$-map.

Remark 1.3. It is important to point out that in general not every $X \in \mathfrak{X}_{\eta}(\mathscr{P})$ corresponds to a Nambu-Poisson system, i.e., a system of the form of equation (1.3). The reason is that the set of vector fields of the form of equation (1.3) is not closed under linear combinations.

There are also fundamental differences between Hamiltonian and Nambu-Poisson mechanics, i.e., between $k=2$ and $k \geq 3$. In particular there is the following result, conjectured by Chatterjee and Takhtajan [4] and later proved by several authors.

Theorem 1.3 ([6, 1, 22, 11, 13]). A totally skew-symmetric contravariant tensor of order $k \geq 3$ is a Nambu-Poisson tensor if and only if it is locally decomposable about any regular point. That is, about any point $\boldsymbol{x} \in \mathscr{P}$ such that $\eta(\boldsymbol{x}) \neq 0$ there exist local coordinates $\left(x_{1}, \ldots, x_{k}, x_{k+1}, \ldots, x_{n}\right)$ such that

$$
\eta=\frac{\partial}{\partial x_{1}} \wedge \cdots \wedge \frac{\partial}{\partial x_{k}}
$$

Thus, every Nambu-Poisson tensor with $k \geq 3$ is in essence a determinant on a sub-manifold of dimension $k$. It is not so for Poisson tensors. 


\subsection{Time transformation of dynamical systems}

In this section we review the time transformation technique developed in Hairer and Söderlind [9]. Consider a dynamical system

$$
\frac{\mathrm{d} \boldsymbol{x}}{\mathrm{d} t}=X(\boldsymbol{x}), \quad X \in \mathfrak{X}(\mathscr{P})
$$

Its flow map is denoted $\varphi_{X}^{t}$. Introduce an extended phase space $\overline{\mathscr{P}}=\mathscr{P} \times \mathbb{R}$, with local coordinates $\overline{\boldsymbol{x}}=(\boldsymbol{x}, \xi)$. The projection $\overline{\mathscr{P}} \ni \overline{\boldsymbol{x}} \mapsto \boldsymbol{x} \in \mathscr{P}$ is denoted $\Pi$, and $\overline{\mathscr{P}} \ni \overline{\boldsymbol{x}} \mapsto \xi \in \mathbb{R}$ is denoted $\pi$. Let $Q \in\{F \in \mathcal{F}(\mathscr{P}) ; F>0\}$ and consider the extension of system (1.5) into

$$
\left\{\begin{array}{ll}
\frac{\mathrm{d} \boldsymbol{x}}{\mathrm{d} \tau}=X(\boldsymbol{x}) / \xi & \text { or shorter }
\end{array} \quad \frac{\mathrm{d} \overline{\boldsymbol{x}}}{\mathrm{d} \xi}=\bar{X}(\overline{\boldsymbol{x}})\right.
$$

The flows of the two systems are related by a reparametrisation $t \leftrightarrow \tau$.

Theorem 1.4 (Hairer and Söderlind [9]). The flow of the extended system (1.6) restricted to $\mathscr{P}$ is a time transformation of the flow of system (1.5). That is,

$$
\Pi \varphi_{\bar{X}}^{\tau}(\overline{\boldsymbol{x}})=\varphi_{X}^{\sigma(\tau, \overline{\boldsymbol{x}})}(\boldsymbol{x}), \quad \forall \overline{\boldsymbol{x}} \in \overline{\mathscr{P}}, \tau \in \mathbb{R} \quad \text { where } \quad \sigma(\tau, \overline{\boldsymbol{x}}) \equiv \int_{0}^{\tau} \frac{\mathrm{d} s}{\pi \varphi_{\bar{X}}^{s}(\overline{\boldsymbol{x}})}
$$

Further, $Q(\boldsymbol{x}) / \xi$ is a first integral of system (1.6).

Proof. From equation (1.6) it follows directly that $\Pi_{*} \bar{X}$ is parallel with $X$. Thus, $\Pi \varphi_{\bar{X}}^{\tau}$ and $\varphi_{X}^{t}$ define the same phase diagrams. It remains to find the relation between $t$ and $\tau$. Since $\mathrm{d} x / \mathrm{d} t=(\mathrm{d} t / \mathrm{d} \tau)(\mathrm{d} x / \mathrm{d} \tau)$ it follows from equation (1.6) that $\mathrm{d} t / \mathrm{d} \tau=1 / \xi$. Integration of this relation gives $\sigma(\tau, \overline{\boldsymbol{x}})$. Further, straightforward calculations and utilisation of the governing equations (1.6) show that $\mathrm{d}(Q(\boldsymbol{x}) / \xi) / \mathrm{d} \tau=0$.

Remark 1.4. It is clear that the time transformation is determined by $Q$. Since $Q$ is strictly positive, the map $\sigma(\cdot, \overline{\boldsymbol{x}}): \mathbb{R} \rightarrow \mathbb{R}$ is bijective, i.e., the reparametrisation $t \leftrightarrow \tau$ is bijective.

In Hairer and Söderlind [9], the motivation for the extended time transformation (1.6) is to construct explicit adaptive numerical integrators for reversible systems. The key is that under reversibility of $Q$, the extended time transformation (1.6) preserves reversibility. First, recall the basic definitions of reversible systems.

Definition 1.2. Let $R \in \operatorname{Diff}(\mathscr{P})$.

- A vector field $X \in \mathfrak{X}(\mathscr{P})$ is called reversible with respect to $R$ if $R_{*} \circ X=-X \circ R$, or equivalently $\mathrm{d}(R(\boldsymbol{x})) / \mathrm{d} t=-(X \circ R)(\boldsymbol{x})$.

- A diffeomorphism $\Phi \in \operatorname{Diff}(\mathscr{P})$ is called reversible with respect to $R$ if $R \circ \Phi=\Phi^{-1} \circ R$.

It is a well known result that the flow of a system is reversible if and only if its corresponding vector field is reversible $[12,8]$. Now, concerning time transformation of reversible systems, it is straightforward to check the following result.

Theorem 1.5 (Hairer and Söderlind [9]). If $X \in \mathfrak{X}(\mathscr{P})$ is reversible with respect to $R$ and $Q \in \mathcal{F}(\mathscr{P})$ fulfils $Q=Q \circ R$, then the vector field $\bar{X} \in \mathfrak{X}(\overline{\mathscr{P}})$ in equation (1.6) is reversible with respect to $\bar{R}: \overline{\boldsymbol{x}} \mapsto(R(\boldsymbol{x}), \xi)$. 


\section{Nambu-Poisson extensions and time transformations}

In this section we develop a time transformation technique for Nambu-Poisson systems. Let $\mathscr{P}$ be a Nambu-Poisson manifold of order $k$ and $\eta$ its Nambu-Poisson tensor. Consider again the extended phase space $\overline{\mathscr{P}}=\mathscr{P} \times \mathbb{R}$. Our first goal is to introduce a Nambu-Poisson structure on $\overline{\mathscr{P}}$. The most natural extension of the Nambu-Poisson tensor $\eta$ is given by

$$
\bar{\eta}=\eta \wedge \frac{\partial}{\partial \xi}
$$

It is not obvious that the bracket corresponding to $\bar{\eta}$ will fulfil the fundamental identity (1.2c). For example, in the canonical Poisson case, i.e., $k=2$, it is not so if $n \geq 3$.

Lemma 2.1. If $k \geq 3$ or $k=n=2$, then $\bar{\eta}$ given by equation (2.1) defines a Nambu-Poisson structure of order $k+1$ on $\overline{\mathscr{P}}$.

Proof. If $k \geq 3$ then it follows from Theorem 1.3 that $\eta$ is decomposable about its regular points, and when $k=n=2$ it is obviously so. Thus, $\eta \wedge \frac{\partial}{\partial \xi}$ is also decomposable about its regular points, so the assertion follows from Theorem 1.3.

The bracket associated with $\bar{\eta}$ is denoted $\overline{\{} \cdot, \ldots, \cdot \overline{\}}$.

Let $H_{1}, \ldots, H_{k-1} \in \mathcal{F}(\mathscr{P})$ be the Hamiltonians for a Nambu-Poisson system on $\mathscr{P}$, i.e., of the form of system (1.3). Further, let $G \in \mathcal{F}(\overline{\mathscr{P}})$ and consider the system on $\overline{\mathscr{P}}$ given by

$$
\frac{\mathrm{d} F}{\mathrm{~d} \tau}=\overline{\{} H_{1}, \ldots, H_{k-1}, G, F \overline{\}}, \quad \forall F \in \mathcal{F}(\overline{\mathscr{P}})
$$

Remark 2.1. A functions $H \in \mathcal{F}(\mathscr{P})$ is considered to belong to $\mathcal{F}(\overline{\mathscr{P}})$ by the natural extension $\overline{\boldsymbol{x}} \mapsto H(\boldsymbol{x})$. Likewise, $\bar{H} \in \mathcal{F}(\overline{\mathscr{P}})$ is considered to be a function in $\mathcal{F}(\mathscr{P})$ depending on the parameter $\xi$. Thus, $\overline{\{} \cdot, \ldots, \cdot \overline{\}}$ is defined also for elements in $\mathcal{F}(\mathscr{P})$ and vice versa.

We continue with the main result in the paper. It states that time transformation of a $\mathrm{Nambu}-\mathrm{Poisson}$ system can be realised as an extended Nambu-Poisson system.

Theorem 2.1. Let $G \in \mathcal{F}(\overline{\mathscr{P}})$ and assume the conditions in Lemma 2.1 are valid. Then:

1. The extended system (2.2) is a Nambu-Poisson system.

2. Its flow restricted to $\mathscr{P}$ is a time transformation, determined by the additional first integral $G$, of the flow of system (1.3). That is,

$$
\Pi \varphi_{H_{1}, \ldots, H_{k-1}, G}^{\tau}(\overline{\boldsymbol{x}})=\varphi_{H_{1}, \ldots, H_{k-1}}^{\sigma(\tau, \bar{x})}(\boldsymbol{x}), \quad \forall \overline{\boldsymbol{x}} \in \overline{\mathscr{P}}, \tau \in \mathbb{R}
$$

where

$$
\sigma(\tau, \overline{\boldsymbol{x}}) \equiv \int_{0}^{\tau} \frac{\partial G}{\partial \xi}\left(\varphi_{H_{1}, \ldots, H_{k-1}, G}^{s}(\overline{\boldsymbol{x}})\right) \mathrm{d} s
$$

Proof. The first assertion follows directly from Lemma 2.1, since $\bar{\eta}$ is a Nambu-Poisson tensor. Since $H_{i}$ for $i=1, \ldots, k-1$ are independent of $\xi$, it follows from the definition (2.1) of $\bar{\eta}$ that

$$
\overline{\{} H_{1}, \ldots, H_{k-1}, G, F \overline{\}}=\frac{\partial G}{\partial \xi}\left\{H_{1}, \ldots, H_{k-1}, F\right\}-\frac{\partial F}{\partial \xi}\left\{H_{1}, \ldots, H_{k-1}, G\right\}
$$


Thus, for $F=x_{1}, \ldots, x_{n}$, the governing equations (2.2) are parallel with those of system (1.3a), i.e., $\Pi \varphi_{H_{1}, \ldots, H_{k-1}, G}^{\tau}$ and $\varphi_{H_{1}, \ldots, H_{k-1}}^{t}$ defined the same phase diagram. The relation between $\tau$ and $t$ is given by $\mathrm{d} t / \mathrm{d} \tau=\partial G / \partial \xi$, which, after integration, gives the desired form of $\sigma(\tau, \overline{\boldsymbol{x}})$.

It is straightforward to check the following corollary, which shows that the technique used by Hairer and Söderlind [9], reviewed in Section 1.2, is a special case.

Corollary 2.1. The case $G(\overline{\boldsymbol{x}})=\log (\xi / Q(\boldsymbol{x}))$ coincides with the transformation (1.6) applied to system (1.3).

\section{Reversible Nambu-Poisson systems}

Recall that the time transformation by Hairer and Söderlind [9] is developed with reversible systems in mind. In the previous section we developed a similar approach, but based on the Nambu-Poisson framework. One may ask under what conditions a Nambu-Poisson system is reversible, and in what sense the time transformation technique developed above preserves reversibility. These questions are studied in this section.

As a first step, we have some results on necessary and sufficient conditions for a NambuPoisson system to be reversible.

Proposition 3.1. Let $R \in \operatorname{Diff}(\mathscr{P})$. Then $X_{H_{1}, \ldots, H_{k-1}}$ is reversible with respect to $R$ if and only if

$$
\left\{H_{1}, \ldots, H_{k-1}, F \circ R\right\}=-\left\{H_{1}, \ldots, H_{k-1}, F\right\} \circ R, \quad \forall F \in \mathcal{F}(\mathscr{P})
$$

Proof. Since $R$ is a diffeomorphism it holds that $\mathcal{F}(\mathscr{P}) \circ R=\mathcal{F}(\mathscr{P})$, so the governing equations (1.3a) are equivalent to

$$
\frac{\mathrm{d}(F \circ R)}{\mathrm{d} t}=\left\{H_{1}, \ldots, H_{k-1}, F \circ R\right\}, \quad \forall F \in \mathcal{F}(\mathscr{P})
$$

This is equivalent to

$$
\frac{\mathrm{d}(F \circ R)}{\mathrm{d} t}=-\left\{H_{1}, \ldots, H_{k-1}, F\right\} \circ R, \quad \forall F \in \mathcal{F}(\mathscr{P})
$$

if and only if condition (3.1) holds. The last set of equations is exactly the condition on $X_{H_{1}, \ldots, H_{k-1}}$ for reversibility with respect to $R$.

If $R$ is a Nambu-Poisson map the assertion may be stated in the following way instead.

Corollary 3.1. Let $R$ be a Nambu-Poisson map, i.e., $R \in \operatorname{Diff}_{\eta}(\mathscr{P})$. Then $X_{H_{1}, \ldots, H_{k-1}}$ is reversible with respect to $R$ if and only if

$$
\left\{H_{1}, \ldots, H_{k-1}, F\right\}=-\left\{H_{1} \circ R, \ldots, H_{k-1} \circ R, F\right\}, \quad \forall F \in \mathcal{F}(\mathscr{P})
$$

Proof. With $F$ set to $F \circ R$, it is clear that the condition (3.2) is equivalent to the condition (3.1) if $R \in \operatorname{Diff}_{\eta}(\mathscr{P})$.

As a generalisation of Theorem 1.5, we now show in what way reversibility of a NambuPoisson system is preserved by the time transformed extended system (2.2). 
Theorem 3.1. Let the system (1.3) be reversible with respect to $R$. Then the extended time transformed Nambu-Poisson system (2.2) is reversible with respect to $\bar{R}: \overline{\boldsymbol{x}} \mapsto(R(\boldsymbol{x}), \xi)$ if $G \circ \bar{R}=G$.

Proof. Since $\partial H_{i} / \partial \xi=0$ we have

$$
\overline{\{} H_{1}, \ldots, H_{k-1}, G, F \circ \bar{R} \overline{\}}=\frac{\partial G}{\partial \xi}\left\{H_{1}, \ldots, H_{k-1}, F \circ \bar{R}\right\}-\frac{\partial(F \circ \bar{R})}{\partial \xi}\left\{H_{1}, \ldots, H_{k-1}, G\right\}
$$

Since $\bar{R}$ maps $\xi$ to $\xi$ it holds that $\partial(F \circ \bar{R}) / \partial \xi=\partial F / \partial \xi \circ \bar{R}$. Further, $G=G \circ \bar{R}$ yields $\partial G / \partial \xi=\partial G / \partial \xi \circ \bar{R}$ and

$$
\left\{H_{1}, \ldots, H_{k-1}, G\right\}=\left\{H_{1}, \ldots, H_{k-1}, G \circ \bar{R}\right\}
$$

Altogether we now have

$$
\begin{aligned}
\left.\overline{\{} H_{1}, \ldots, H_{k-1}, G, F \circ \bar{R}\right\} & =\frac{\partial G}{\partial \xi} \circ \bar{R}\left\{H_{1}, \ldots, H_{k-1}, F \circ \bar{R}\right\}-\frac{\partial F}{\partial \xi} \circ \bar{R}\left\{H_{1}, \ldots, H_{k-1}, G \circ \bar{R}\right\} \\
& =-\frac{\partial G}{\partial \xi} \circ \bar{R}\left\{H_{1}, \ldots, H_{k-1}, F\right\} \circ \bar{R}+\frac{\partial F}{\partial \xi} \circ \bar{R}\left\{H_{1}, \ldots, H_{k-1}, G\right\} \circ \bar{R} \\
& \left.=-\overline{\{} H_{1}, \ldots, H_{k-1}, G, F\right\} \circ \bar{R}
\end{aligned}
$$

where the stipulation that system (1.3) is reversible have been used in conjunction with Proposition 3.1. Application of Proposition 3.1 again completes the assertion.

\section{Application: numerical integration by splitting}

The main motivation for extended time transformations is to construct adaptive numerical integration algorithms. By a numerical integrator for a dynamical system $X \in \mathfrak{X}(\mathscr{P})$, we mean a family of near identity maps $\Phi_{h} \in \operatorname{Diff}(\mathscr{P})$, such that $\Phi_{h}$ is an approximation of the exact flow $\varphi_{X}^{h}$. Numerical solution "paths" are obtained by the discrete dynamical system $\boldsymbol{x}_{k+1}=$ $\Phi_{h}\left(\boldsymbol{x}_{k}\right)$. The integrator $\Phi_{h}$ is consistent of order $p$ if $\Phi_{h}-\varphi^{h}=\mathcal{O}\left(h^{p}\right)$, which in particular implies $\Phi_{0}=$ Id. It is explicit if $\Phi_{h}(\boldsymbol{x})$ can be computed by a finite algorithm. Notice that $\Phi_{h}$ is not a one parameter group, i.e., $\Phi_{h} \circ \Phi_{s} \neq \Phi_{h+s}$.

When constructing numerical integrators, one typically tries to preserve as much as possible of the underlying qualitative structure of the exact flow. In our case, we like $\Phi_{h}$ to preserve the Nambu-Poisson structure, and in presence also reversibility. In addition, time step adaptivity is crucial in order for the integration method to be computationally efficient. Indeed, we would like to vary the step size $h$ during the integration process according to the present local character of the dynamics, without destroying the structural properties of the method. The standard approach, motivating our work, is to utilise a time transformation $t \leftrightarrow \tau$ that preserves the structure of the original system, and then construct a $\tau$-equidistant numerical integrator for transformed system. An equivalent view point is to say that the time transformation should regularise the problem, so that it becomes easier to integrate numerically.

Splitting is a compelling technique for the construction of structure preserving integrators [17]. The basic idea is as follows. Let $\mathfrak{X}_{A}(\mathscr{P})$ be a Lie sub-algebra of $\mathfrak{X}(\mathscr{P})$, and let $\operatorname{Diff}_{A}(\mathscr{P})$ be the corresponding Lie sub-group of $\operatorname{Diff}(\mathscr{P})$. Assume that $X \in \mathfrak{X}_{A}(\mathscr{P})$ can be splitted into explicitly 
integrable sub-system, each of which is a system in $\mathfrak{X}_{A}(\mathscr{P})$. That is, $X=X_{1}+\ldots+X_{k}$, where $X_{i} \in \mathfrak{X}_{A}(\mathscr{P})$ and $\varphi_{X_{i}}^{t}(\boldsymbol{x})$ can be computed explicitly. A numerical integrator for $X$ is obtained by $\Phi_{h}=\varphi_{X_{1}}^{h} \circ \cdots \circ \varphi_{X_{k}}^{h}$. It is clear that $\Phi_{h}$ is an approximation of $\varphi_{X}^{h}$, and that $\Phi_{h} \in \operatorname{Diff}_{A}(\mathscr{P})$. Further, by the Baker-Campbell-Hausdorff (BCH) formula, it follows that $\Phi_{h}$ is the exact flow of a modified vector field $\tilde{X}_{h} \in \mathfrak{X}_{A}(\mathscr{P})$, i.e., $\Phi_{h}=\varphi_{\tilde{X}_{h}}^{h}$. This information is crucial for the analysis of $\Phi_{h}$. For example, if $\mathfrak{X}_{A}(\mathscr{P})$ is the Lie-algebra corresponding to a Poisson structure on $\mathscr{P}$, then $\Phi_{h}$ will exactly conserve a modified Hamiltonian, which is $\mathcal{O}\left(h^{p}\right)$-close to the Hamiltonian for the original problem [8].

Remark 4.1. Due to convergence issues, the $\mathrm{BCH}$ formula needs to be truncated, which implies that assertions on $\Phi_{h}$, coming from $\tilde{X}$, are valid only for exponentially long times, i.e., up to time scales of order $\mathcal{O}\left(\exp \left(\mathcal{O}\left(1 / h^{p}\right)\right)\right)$. See Hairer et. al. [8] for details.

Our notion for the construction of integrators is to utilise the results in Section 2-3, and consider splitting of the individual Nambu-Poisson Hamiltonians.

Let $\eta$ be a Nambu-Poisson tensor. The set of Nambu-Poisson maps which are reversible with respect to $R$ is denoted $\operatorname{Diff}_{\eta, R}(\mathscr{P})$. If $\Phi, \Psi \in \operatorname{Diff}_{\eta, R}(\mathscr{P})$, then in general we have

$$
R \circ \Phi \circ \Psi=\Phi^{-1} \circ R \circ \Psi=\Phi^{-1} \circ \Psi^{-1} \circ R \neq(\Phi \circ \Psi)^{-1} \circ R
$$

Thus, $\operatorname{Diff}_{\eta, R}(\mathscr{P})$ is not closed under composition, so it is not a sub-group of $\operatorname{Diff}(\mathscr{P})$. However, $\operatorname{Diff}_{\eta, R}(\mathscr{P})$ is closed under the symmetric group operation $(\Phi, \Psi) \mapsto \sqrt{\Phi} \circ \Psi \circ \sqrt{\Phi}$, which we write as $\Phi \odot \Psi$. Further, from the symmetric BCH formula (cf. [15]) it follows that if $X, Y \in \mathfrak{X}_{\eta, R}(\mathscr{P})$, then the vector field $Z$ such that $\varphi_{Z}^{t}=\varphi_{X}^{t} \odot \varphi_{Y}^{t}$ belongs to $\mathfrak{X}_{\eta, R}(\mathscr{P})$.

Remark 4.2. For near identity maps, $\sqrt{\Phi}$ is defined by taking its representation $\Phi=\exp (X)$ and then setting $\sqrt{\Phi}=\exp (X / 2)$. In our case, $\Phi$ will always be an exact flow $\varphi_{X}^{t}$, in which case $\sqrt{\varphi_{X}^{t}}=\varphi_{X}^{t / 2}$.

We now give a result concerning reversible systems, which is of use for the analysis of periodic numerical paths of reversible splitting methods.

Lemma 4.1. Let $X \in \mathfrak{X}(\mathscr{P})$ be reversible with respect to $R \in \operatorname{Diff}(\mathscr{P})$. Assume that the set $\mathrm{U}=\{\boldsymbol{x} \in \mathscr{P} ; R(\boldsymbol{x})=\boldsymbol{x}\}$ of fix-points of $R$ is non-empty and that $\gamma: \mathbb{R} \rightarrow \mathscr{P}$ is a solution curve of $X$ for which there exists $t_{1}, t_{2} \in \mathbb{R}$ with $t_{1}<t_{2}$ such that $\gamma\left(t_{1}\right), \gamma\left(t_{2}\right) \in \mathrm{U}$. Then $\gamma$ is periodic.

Proof. For simplicity assume that $t_{1}=0$ and $t_{2}>0$, which is not a restriction. The curve $\gamma_{2}(t)=(R \circ \gamma)(-t)$ is also a solution curve due to reversibility. Further, since $R$ restricted to $U$ is the identity map we have the equalities $\gamma\left(t_{1}\right)=\gamma_{2}\left(t_{1}\right)$ and $\gamma\left(-t_{2}\right)=\gamma_{2}\left(t_{2}\right)$. Due to uniqueness of solutions the first equality implies $\gamma_{2}=\gamma$, which in conjunction with the second equality implies that $\gamma\left(-t_{2}\right)=\gamma\left(t_{2}\right)$. Thus $\gamma$ returns to the same point twice, so it is periodic.

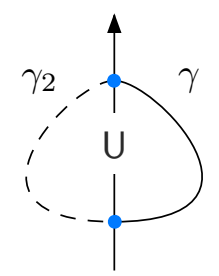

\subsection{Rigid body problem}

The Euler equations for the free rigid body is a Nambu-Poisson system on the phase space $\mathbb{R}^{3}$, equipped with the canonical Nambu-Poisson structure $\eta=\partial / \partial x_{1} \wedge \partial / \partial x_{2} \wedge \partial / \partial x_{3}$. Its two 
Hamiltonians are total angular momentum $M(\boldsymbol{x})=\sum x_{i}^{2} / 2$ and kinetic energy $T(\boldsymbol{x})=\sum x_{i}^{2} /\left(2 I_{i}\right)$, where $I_{i}>0$ are the principal moments of inertia. Thus, the governing equations are

$$
\frac{\mathrm{d} F}{\mathrm{~d} t}=\{M, T, F\}, \quad \forall F \in \mathcal{F}\left(\mathbb{R}^{3}\right)
$$

which explicitly reads

$$
\begin{array}{ll}
\dot{x}_{1}=a_{1} x_{2} x_{3}, & a_{1}=\left(I_{2}-I_{3}\right) /\left(I_{2} I_{3}\right) \\
\dot{x}_{2}=a_{2} x_{3} x_{1}, & a_{2}=\left(I_{3}-I_{1}\right) /\left(I_{3} I_{1}\right) \\
\dot{x}_{3}=a_{3} x_{1} x_{2}, & a_{3}=\left(I_{1}-I_{2}\right) /\left(I_{1} I_{2}\right)
\end{array}
$$

It is straightforward to check that the system is reversible with respect to the linear diffeomorphism $R_{1}: \boldsymbol{x} \mapsto\left(-x_{1}, x_{2}, x_{3}\right)$, and in symmetry, also with respect to $R_{2}, R_{3}$ defined analogously. Thus, due to Lemma 4.1, we have the following KAM-like result for the free rigid body.

Theorem 4.1. Let $\tilde{X}_{h} \in \mathfrak{X}\left(\mathbb{R}^{3}\right)$ depend smoothly on $h$ such that $\tilde{X}_{0}=X_{M, T} \neq 0$. Assume that $\tilde{X}_{h}$, for each $h$, is reversible with respect to $R_{1}, R_{2}$ and $R_{3}$. Then, for small enough $h$, the solution paths of $\tilde{X}_{h}$ are periodic.

Proof. It is known that if $\gamma$ is a solution curve of the Euler equations, then it is either an equilibrium, or it is periodic with finite period $t_{e}>0$, in which case it crosses either of the planes $\mathrm{U}_{\mathrm{i}}=\left\{\boldsymbol{x} \in \mathbb{R}^{3} ; R_{i}(\boldsymbol{x})=\boldsymbol{x}\right\}$ every half period [14]. That is, it holds that $\gamma\left(t_{1}\right), \gamma\left(t_{1}+t_{e} / 2\right) \in \mathrm{U}_{k}$ for some $k \in\{1,2,3\}$ and $t_{1} \in\left[0, t_{e} / 2\right)$. Further, since $X_{M, T} \equiv 0$ is not allowed, it is known that if $\gamma$ is an equilibrium, then $\gamma(t) \in \mathrm{U}_{k}$. Let $\tilde{\gamma}_{h}$ be a solution curve of $\tilde{X}_{h}$ and let $\gamma$ be the solution curve of $X_{M, T}$ such that $\gamma(0)=\tilde{\gamma}_{h}(0)$. Assume first that $\gamma$ is not an equilibrium. Then, for any $\delta \in\left(0, t_{e} / 2\right)$ it holds that a continuous path between $\gamma\left(t_{1}-\delta\right)$ and $\gamma\left(t_{1}+\delta\right)$ must cross the plane $\mathrm{U}_{k}$. For small enough $h$ it holds that $\tilde{\gamma}_{h}\left(t_{1}-\delta\right)$ and $\tilde{\gamma}_{h}\left(t_{1}+\delta\right)$ approximates $\gamma\left(t_{1}-\delta\right)$ and $\gamma\left(t_{1}+\delta\right)$ well enough to also be separated by $\mathrm{U}_{k}$. Thus, $\tilde{\gamma}_{h}\left(\tilde{t}_{1}\right) \in \mathrm{U}_{1}$ for some $\tilde{t}_{1} \in\left(t_{1}-\delta, t_{1}+\delta\right)$. Likewise, $\tilde{\gamma}_{h}\left(\tilde{t}_{2}\right) \in \mathrm{U}_{1}$ for some $\tilde{t}_{2} \in\left(t_{1}+t_{e} / 2-\delta, t_{1}+t_{e} / 2+\delta\right)$. Since $\tilde{X}_{h}$ is reversible with respect to $R_{1}$ it follows from Lemma 4.1 that $\tilde{\gamma}_{h}$ is periodic. If $\gamma$ is an equilibrium and $\tilde{\gamma}_{h}$ is not, then either there exists $s>0$ such that $\tilde{\gamma}_{h}(s) \notin \mathrm{U}_{k}$, in which case the solution curve of $X_{M, T}$ such that $\gamma(s)=\tilde{\gamma}_{h}(s)$ is periodic, so we are back to the first case, or $\tilde{\gamma}_{h}(0), \tilde{\gamma}_{h}(s) \in \mathrm{U}_{k}$, in which case the assertion follows directly from Lemma 4.1 .

The traditional perception in the literature is to view the rigid body equations (4.1) as a Poisson system, with the non-canonical Poisson tensor $\eta_{M}=\eta\left(\mathrm{d} M, \cdot \cdot_{1}, \cdot_{2}\right)$, induced by the total angular momentum ( $M$ is a Casimir, cf. [14], for this Poisson structure). We denote the corresponding bracket by $\left\{\cdot_{1}, \cdot_{2}\right\}_{M}$. It is clear that Diff $\eta_{M}$ is a sub-group of Diff $\eta_{\eta}$. Consider the Hamiltonian splitting $T=\sum T_{i}$, where $T_{i}(\boldsymbol{x})=x_{i}^{2} /\left(2 I_{i}\right)$. The sub-system $\dot{F}=\left\{T_{i}, F\right\}_{M}$ does not affect $x_{i}$, i.e., $\dot{x}_{i}=0$, and all the quadratic terms contain $x_{i}$. Hence, it is in essence a linear system on $\mathbb{R}^{2}$, and therefore explicitly integrable (since the exponential map is computable for any $2 \times 2$-matrix). A well known second order integrator is obtained by the symmetric composition

$$
\Phi_{h}^{\Upsilon T}=\varphi_{M, T_{1}}^{h} \odot \varphi_{M, T_{2}}^{h} \odot \varphi_{M, T_{3}}^{h}
$$

This integrator has the following properties: 
1. It is reversible with respect to $R_{1}, R_{2}$ and $R_{3}$. Thus, its modified vector field $\tilde{X}_{h}$ is a $R_{1}, R_{2}, R_{3}$-reversible perturbation of $X$, so Theorem 4.1 may be used to deduce periodic orbits of the numerical solution.

2. It is a Poisson map, i.e., $\Phi_{h}^{T} \in \operatorname{Diff}_{\eta_{M}}(\mathscr{P})$. This implies that its modified vector field $\tilde{X}_{h}$ is the Hamiltonian vector field of a modified Hamiltonian $\tilde{T}_{h}=T+\mathcal{O}\left(h^{2}\right)$, so $T$ is nearly conserved. Further, since $M$ is a Casimir of the Poisson structure it is exactly conserved.

Remark 4.3. One may also view the rigid body equations (4.1) as a Poisson system with the Poisson tensor $\eta_{T}=\eta\left(\cdot \cdot_{1}, \mathrm{~d} T, \cdot_{2}\right)$, and then construct an integrator $\Phi_{h}^{\Upsilon M}$ by splitting of $M$. This integrator will exactly conserve $T$, and nearly conserve $M$.

Following our notion, we now consider Hamiltonian splitting of both $M$ and $T$. To this end, let $M_{i}(\boldsymbol{x})=x_{i}^{2} / 2$. Since $X_{M_{i}, T_{i}}=0$ it follows that

$$
X_{M, T}=X_{M_{1}+M_{2}, T_{1}+T_{2}}+X_{M_{2}+M_{3}, T_{2}+T_{3}}+X_{M_{3}+M_{1}, T_{3}+T_{1}}
$$

Each such vector field is integrable by linear extrapolation, for example,

$$
\varphi_{M_{1}+M_{2}, T_{1}+T_{2}}^{t}(\boldsymbol{x})=\boldsymbol{x}+t X_{M_{1}+M_{2}, T_{1}+T_{2}}(\boldsymbol{x})
$$

Thus, a second order integrator is obtained by

$$
\Phi_{h}^{\curlyvee M^{\curlyvee} T}=\varphi_{M_{1}+M_{2}, T_{1}+T_{2}}^{h} \odot \varphi_{M_{2}+M_{3}, T_{2}+T_{3}}^{h} \odot \varphi_{M_{3}+M_{1}, T_{3}+T_{1}}^{h}
$$

This integrator is computationally cheaper than $\Phi_{h}^{\Upsilon T}$, since computation of the exponential map, which involves evaluation of sin and cos, is not necessary. Further, it has the following properties:

1. It is reversible with respect to $R_{1}, R_{2}$ and $R_{3}$. Thus, its modified vector field $\tilde{X}_{h}$ is a $R_{1}, R_{2}, R_{3}$-reversible perturbation of $X_{M, T}$, so Theorem 4.1 may be used to deduce periodic orbits of the numerical solution.

2. It is an $\eta$-map, i.e., $\Phi_{h}^{\curlyvee M^{\curlyvee} T} \in \operatorname{Diff}_{\eta}$, which implies $\tilde{X} \in \mathfrak{X}_{\eta}(\mathscr{P})$. However, $\Phi_{h}^{\curlyvee} M^{\curlyvee} T$ does not correspond to a modified Nambu-Poisson system (see Remark 1.3), so there are no exactly conserved modified Hamiltonians $\tilde{M}$ and $\tilde{T}$. Nevertheless, $M$ and $T$ are still nearly conserved due to the periodicity of the numerical solution.

Consider now time transformation of system (4.1) into an extended Nambu-Poisson system

$$
\frac{\mathrm{d} F}{\mathrm{~d} \tau}=\overline{\{} M, T, G, F \overline{\}}, \quad \forall F \in \mathcal{F}\left(\mathbb{R}^{4}\right)
$$

We have the following generalisation of Theorem 4.1.

Theorem 4.2. Let $\tilde{X}_{\epsilon} \in \mathfrak{X}\left(\mathbb{R}^{4}\right)$ depend smoothly on $\epsilon$ such that $\tilde{X}_{0}=X_{M, T, G} \neq 0$. Assume that $\tilde{X}_{\epsilon}$, for each $\epsilon$, is reversible with respect to $\bar{R}_{1}, \bar{R}_{2}$ and $\bar{R}_{3}$, and that there exists $\delta>0$ such that $\partial G / \partial \xi>\delta$. Then, for small enough $\epsilon$, the solution paths of $\tilde{X}_{\epsilon}$ are periodic.

Proof. From the definition of $\bar{R}_{i}$ it follows that $\bar{U}_{i}=\left\{\overline{\boldsymbol{x}} \in \mathbb{R}^{4} ; \bar{R}_{i}(\overline{\boldsymbol{x}})=\overline{\boldsymbol{x}}\right\}$ is a hyper-plane, and that $\mathbb{R}^{3} \ni \boldsymbol{x} \in \mathrm{U}_{i}$ implies $(\boldsymbol{x}, \xi) \in \overline{\mathbf{U}}_{i}$ for all $\xi \in \mathbb{R}$. Let $\gamma$ be a solution curve of $X_{M, T, G}$. Since it is a time transformation of a solution curve of $X_{M, T}$ and since $\partial G / \partial \xi>\delta$ it follows that there exists $t_{1}<t_{2}$ and $k \in\{1,2,3\}$ such that $\gamma\left(t_{1}\right), \gamma\left(t_{2}\right) \in \overline{\mathbf{U}}_{k}$. Thus, $\gamma$ is periodic due to Lemma 4.1. The proof now proceeds exactly as the proof of Theorem 4.1. 
Assume $G$ takes the splitted form $G(\overline{\boldsymbol{x}})=G_{1}(\boldsymbol{x})+G_{2}(\xi)$. We propose the following adaptive versions of $\Phi_{h}^{\curlyvee}$ and $\Phi_{h}^{\curlyvee} M^{\curlyvee} T$.

$$
\begin{aligned}
\Phi_{\epsilon}^{\curlyvee} T^{\curlyvee} G & =\varphi_{M, T, G_{1}}^{\epsilon} \odot \varphi_{M, T_{1}, G_{2}}^{\epsilon} \odot \varphi_{M, T_{2}, G_{2}}^{\epsilon} \odot \varphi_{M, T_{3}, G_{2}}^{\epsilon} \\
\Phi_{\epsilon}^{\curlyvee} M^{\curlyvee} T^{\curlyvee} G & =\varphi_{M, T, G_{1}}^{\epsilon} \odot \varphi_{M_{1}+M_{2}, T_{1}+T_{2}, G_{2}}^{\epsilon} \varphi_{M_{2}+M_{3}, T_{2}+T_{3}, G_{2}}^{\epsilon} \odot \varphi_{M_{3}+M_{1}, T_{3}+T_{1}, G_{2}}^{\epsilon}
\end{aligned}
$$

Notice that all of the partial flows are explicitly integrable. In particular, $\varphi_{M, T, G_{1}}^{\epsilon}(\overline{\boldsymbol{x}})=\overline{\boldsymbol{x}}+$ $\epsilon X_{M, T, G_{1}}(\overline{\boldsymbol{x}})$. Further, it holds that

$$
\varphi_{M, T_{i}, G_{2}}^{\epsilon}(\overline{\boldsymbol{x}})=\left(\varphi_{M, T_{i}}^{\epsilon \frac{\partial G_{2}}{\partial \xi}(\xi)}(\boldsymbol{x}), 0\right), \quad i=1,2,3
$$

and correspondingly for $\varphi_{M_{i}, T_{i}, G_{2}}^{\epsilon}$. These integrators have the following properties:

1. They are reversible with respect to $\bar{R}_{1}, \bar{R}_{2}$ and $\bar{R}_{3}$. Thus, their modified vector fields are $\bar{R}_{1}, \bar{R}_{2}, \bar{R}_{3}$-reversible perturbation of $X_{M, T, G}$, so Theorem 4.2 may be used to deduce periodic orbits of the numerical solution. (Assuming $\exists \varepsilon>0$ such that $\partial G_{2} / \partial \xi>\varepsilon$.)

2. They are $\bar{\eta}-$ maps. However, they do not correspond to a modified Nambu-Poisson system (see Remark 1.3). Nevertheless, $M, T$ and $G$ are still nearly conserved due to the periodicity of the numerical solution. In fact, $M$ is exactly conserved by $\Phi^{\curlyvee} T^{\curlyvee} G$, since each partial flow is an $\eta_{M}-$ map.

As an illustration, numerical simulations with $\Phi^{\curlyvee M^{\curlyvee} T}, \Phi^{\curlyvee} T, \Phi^{\curlyvee} M^{\curlyvee} T^{\curlyvee} G$, and $\Phi^{\curlyvee} T^{\curlyvee} G$ are given. The moments of inertia are $I_{1}=1 / 2, I_{2}=1, I_{3}=2$, and initial data are $\boldsymbol{x}_{0}=(0, \cos (\theta), \sin (\theta))$, with $\theta=0.2$, which correspond to rotation "nearly" about the unstable principle axis. For the adaptive integrators the additional Hamiltonian is $G=G_{1}+G_{2}=-\log \left(\left\|X_{M, T}\right\|+0.01\right)+\log (\xi)$, so the steps become smaller as the magnitude of the vector field $X_{M, T}$ increases. The step size $h=0.15$ is used for the non-adaptive integrators, and for the adaptive integrators $\epsilon$ is chosen to yield the same mean time step (i.e. so that the mean of $\epsilon \partial G / \partial \xi$ is $h$ ).

A comparison of solutions in the $t$ (non-adaptive) and in the $\tau$ (adaptive) variables are given in Figure 1. Notice that the time-stretching makes the solution "smoother". Further, the numerical errors in the Hamiltonians are plotted in Figure 2. Notice that the errors are significantly smaller for the adaptive integrators.

\section{Conclusions}

A time transformation technique for Nambu-Poisson systems, based on extending the phase space, have been developed (Theorem 2.1). The technique is shown to preserve reversibility under mild conditions on the additional Hamiltonian function (Theorem 3.1). A family of numerical integrators based on splitting of the Nambu-Poisson Hamiltonians is suggested. In particular, a novel approach for numerical integration of the Euler equations for the free rigid body is presented. By backward error analysis, it is shown that periodicity is preserved (Theorem 4.1 and Theorem 4.2).

Acknowledgement The author is grateful to Claus Führer, Gustaf Söderlind and Sergei Silvestrov for fruitful discussions, and to Olivier Verdier for many helpful suggestions on improvement. The author would also like to thank SKF for the support given. 


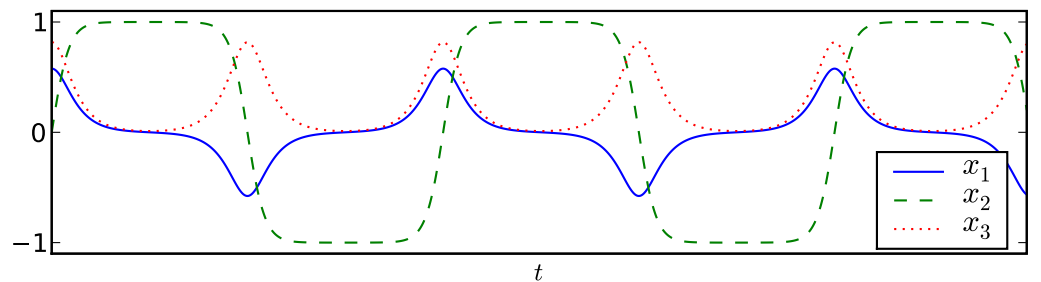

$$
\Phi_{h}^{\curlyvee} T
$$

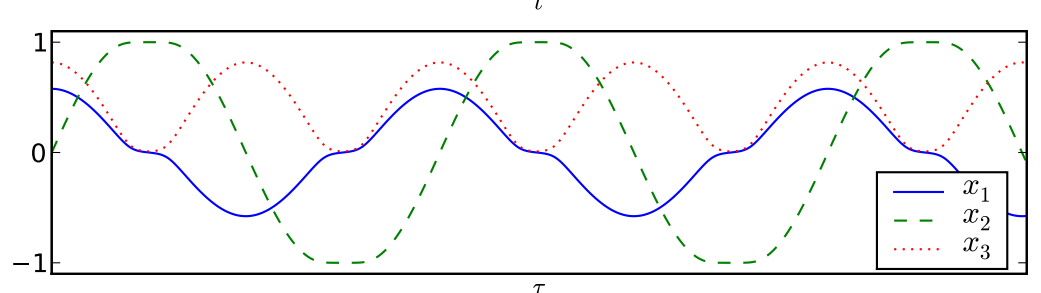

$\Phi_{h}^{\curlyvee} T^{\curlyvee} G$

Figure 1. Solution curves for the non-adaptive integrator $\Phi^{\curlyvee} T$, and for the adaptive integrator $\Phi^{\curlyvee} T^{\curlyvee} G$. Notice that the curves in the lower graph, corresponding to $\Phi^{\curlyvee} T^{\curlyvee} G$, are "smoother". This is due to the time-stretching.

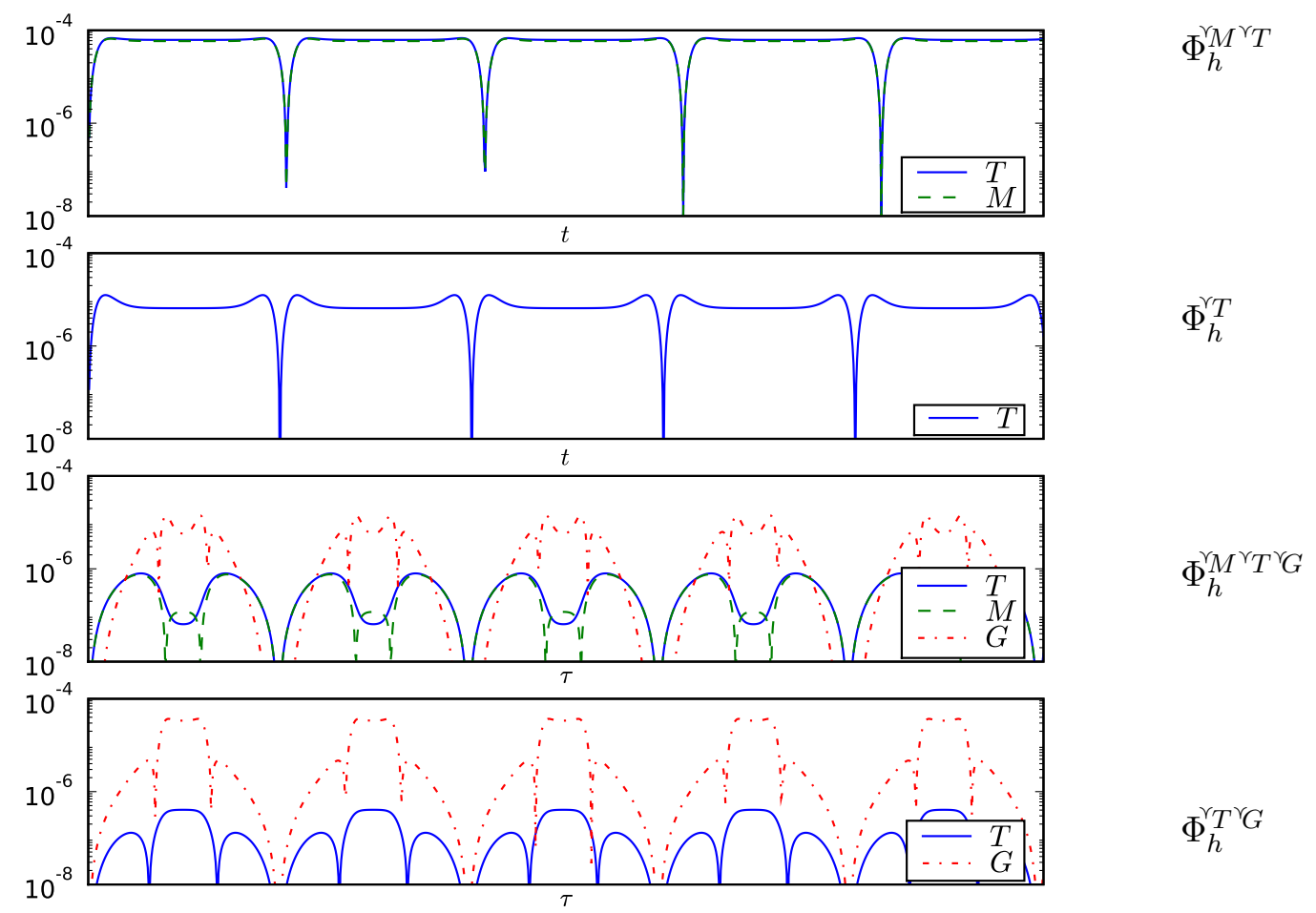

Figure 2. Absolute errors in the Hamiltonians. Notice that the errors in $T$ (and $M$ ) are significantly smaller for the adaptive integrators. Thus, increased efficiency due to adaptivity is obtained. (Recall that $\Phi^{\curlyvee} T$ and $\Phi^{\curlyvee} T^{\curlyvee} G$ conserve $M$ up to rounding errors, whence $M$ is not plotted for these.) 


\section{References}

[1] D. Alekseevsky and P. Guha. On decomposability of Nambu-Poisson tensor. Acta Math. Univ. Comenian. (N.S.), 65 (1996), 1-9.

[2] S. Blanes and C. J. Budd. Adaptive geometric integrators for Hamiltonian problems with approximate scale invariance. SIAM J. Sci. Comput., 26 (2005), 1089-1113 (electronic).

[3] S. D. Bond and B. J. Leimkuhler. Time-transformations for reversible variable stepsize integration. Numer. Algorithms, 19 (1998), 55-71,

[4] R. Chatterjee and L. Takhtajan. Aspects of classical and quantum Nambu mechanics. Lett. Math. Phys. 37 (1996), 475-482.

[5] S. Codriansky, R. Navarro, and M. Pedroza. The Liouville condition and Nambu mechanics. J. Phys. A, 29 (1996), 1037-1044.

[6] P. Gautheron. Some remarks concerning Nambu mechanics. Lett. Math. Phys. 37 (1996),103-116.

[7] E. Hairer. Variable time step integration with symplectic methods. Appl. Numer. Math., 25 (1007), 219-227.

[8] E. Hairer, C. Lubich, and G. Wanner. Geometric numerical integration. Springer Series in Computational Mathematics, 31, Springer-Verlag, Berlin, Second Ed., 2006.

[9] E. Hairer and G. Söderlind. Explicit, time reversible, adaptive step size control. SIAM J. Sci. Comput. 26 (2005), 1838-1851 (electronic).

[10] W. Huang and B. Leimkuhler. The adaptive Verlet method. SIAM J. Sci. Comput. 18 (1997), 239-256.

[11] R. Ibáñez, M. de León, J. C. Marrero, and D. Martin de Diego. Dynamics of generalized Poisson and Nambu-Poisson brackets. J. Math. Phys. 38 (1997), 2332-2344.

[12] J. S. W. Lamb and J. A. G. Roberts. Time-reversal symmetry in dynamical systems: a survey. Phys. D, 112 (1998), 1-39.

[13] G. Marmo, G. Vilasi, and A. M. Vinogradov. The local structure of $n$-Poisson and $n$-Jacobi manifolds. J. Geom. Phys. 25 (1998), 141-182.

[14] J. E. Marsden and T. S. Ratiu. Introduction to mechanics and symmetry, Texts in Applied Mathematics, 17, Springer-Verlag, New York, Second Ed., 1999.

[15] R. I. McLachlan. On the numerical integration of ordinary differential equations by symmetric composition methods. SIAM J. Sci. Comput. 16 (1995),151-168.

[16] R. I. McLachlan and G. R. W. Quispel. What kinds of dynamics are there? Lie pseudogroups, dynamical systems and geometric integration. Nonlinearity, 14 (2001), 1689-1705.

[17] R. I. McLachlan and G. R. W. Quispel. Splitting methods. Acta Numer. 11 (2002), 341-434, 2002.

[18] S. Mikkola and P. Wiegert. Regularizing time transformations in symplectic and composite integration. Celestial Mech. Dynam. Astronom. 82 (2002), 375-390.

[19] K. Modin. On explicit adaptive symplectic integration of separable Hamiltonian systems. Journal of Multibody Dynamics, 2008 (to be published).

[20] K. Modin and C. Führer. Time-step adaptivity in variational integrators with application to contact problems. ZAMM Z. Angew. Math. Mech. 86 (2006), 785-794.

[21] P. Morando. Liouville condition, Nambu mechanics, and differential forms. J. Phys. A, 29 (1996), L329-L331.

[22] N. Nakanishi. On Nambu-Poisson manifolds. Rev. Math. Phys. 10 (1998), 499-510.

[23] Y. Nambu. Generalized Hamiltonian dynamics. Phys. Rev. D, 7 (1973), 2405-2412.

[24] M. Preto and S. Tremaine. A class of symplectic integrators with adaptive timestep for separable hamiltonian systems. Astron. J. 118 (1999), 2532-2541.

[25] R. Schmid. Infinite dimensional Lie groups with applications to mathematical physics. J. Geom. Symmetry Phys. 1 (2004), 54-120.

[26] D. Stoffer. Variable steps for reversible integration methods. Computing, 55 (1995), 1-22. 
[27] L. Takhtajan. On foundation of the generalized Nambu mechanics. Comm. Math. Phys. 160 (1994), $295-315$.

[28] I. Vaisman. A survey on Nambu-Poisson brackets. Acta Math. Univ. Comenian. (N.S.), 68 (1999), $213-241$.

[29] I. Vaisman. Nambu-Lie groups. J. Lie Theory, 10 (2000), 181-194.

Received September 10, 2008

Revised October 07, 2008 